\title{
ELEMENTARY GROUPS
}

\author{
BY \\ HOMER BECHTELL
}

1. Introduction. The purpose of this paper is to investigate two classes of finite groups, elementary groups and $E$-groups. The elementary groups have the property that the Frattini subgroup, $\Phi(G)$, of the group $G$ is the identity and each element $x \in G$ is not only a generator of $G$, but also of each subgroup of $G$ containing $x$. The structure of these groups is fully developed. Moreover, the elementary groups provide the first "large" class of groups, whose structure is known, having the property that each subgroup of the group is complemented ( $K$-groups). From these groups one proceeds to a class of groups $G$ having the property that $G / \Phi(G)$ is an elementary group. They are designated as $E$-groups and $\Phi(H) \leqq \Phi(G)$ for each subgroup $H$ of $G$. Since all nilpotent groups have this property, the class is not empty.

The paper ends with a discussion of a unique characteristic subgroup, $E(G)$, of an arbitrary group $G$ called the elementary commutator of $G$. $G / E(G)$ is an elementary group and $E(G)$ is contained in any normal subgroup $N$ of $G$ for which $G / N$ is elementary.

Numbers in brackets refer to the bibliography. Unless indicated, the notation and the definitions used will be those found in the standard references such as $M$. Hall [4]. The alternative definitions and the basic properties of the Frattini subgroup will be assumed known to the reader. Only finite groups will be considered.

This paper is a portion of the author's doctoral dissertation at the University of $W$ isconsin. He expresses his sincere appreciation for the encouragement given by Professor R. H. Bruck who directed the study.

2. Elementary groups. If for a normal subgroup $N$ of a group $G$ there exists a proper subgroup $H$ of $G$ such that $G=N H$, then $G$ is said to be the partial product of $N$ by $H$. It is clear that for each partial product of $N$ by $H$ there is a reduced partial product, i.e., one in which for no proper subgroup $C$ of $H$ does $G=N C$. Consequently, in a reduced partial product $G=N H, N$ normal in $G$, it is seen that $N \cap H \leqq \Phi(H)$. For those groups $G$ having $\Phi(G)=1$, the $\Phi$-free groups, $G$ may be expressed as a reduced partial product over each of its normal subgroups. However, this condition alone does not imply that each such product is a semi-direct product. This can be overcome by insisting that each subgroup of $G$ is $\Phi$-free.

Presented to the Society, May 9, 1962, under the title On finite groups whose generators are subgroup generators. II; received by the editors December 17, 1963. 
Definition. A group $G$ is an elementary group provided that $\Phi(H)=1$ for each subgroup $H \leqq G$.

The most well-known elementary groups are the elementary Abelian groups. However, the symmetric group on three symbols and the alternating group on five symbols exemplify, respectively, the existence of solvable and simple elementary groups.

The property is clearly hereditary, the elements are of square-free order, and each element is not only a group generator but also a generator of each subgroup that contains it.

Consider now the subgroup lattice of an elementary group. A group is called a $K$-group provided that the subgroup lattice is complemented (see Suzuki [6, p. 26]). In general the structure of $K$-groups is still an open question and except for the supersolvable $K$-groups, very little at all is known about them.

Lemma 2.1. An elementary group is a $K$-group.

Proof. If for a subgroup $H$ of an elementary group $G, H=1$ or $G$, then clearly there is a complement; so assume that $H$ is neither of these. $\Phi(G)=1$ implies the existence of a proper subgroup $K$ of $G$ such that $G=\{H, K\}$. Suppose $H \cap K \neq 1$. Then $\Phi(K)=1$ implies there exists a proper subgroup $K_{1}$ of $K$ such that $K=\left\{H \cap K, K_{1}\right\}$ or $G=\left\{H, H \cap K, K_{1}\right\}=\left\{H, K_{1}\right\}$. Continuing in this manner, one arrives at an integer $n$ for which $G$ $=\left\{H, K_{n}\right\}, H \cap K_{n}=1$.

COROLlary 2.1. An elementary group splits over each of its normal subgroups.

The symmetric group on four symbols, $S_{4}$, provides a counterexample to the converse of the corollary. $S_{4}$ is a $K$-group but not an elementary group since it contains an element of order four. Thus in general the property of being a $K$-group is not hereditary and so the elementary groups can form only a proper subset of the set of all $K$-groups.

TheOREM 2.2. $G$ is an elementary group if and only if

(i) $G$ is a $K$-group and

(ii) each subgroup of $G$ is a $K$-group.

Theorem 2.3. For a group $G$ the following statements are equivalent:

(i) If $H$ is a subgroup of $G$ and $N$ is a normal subgroup of $H$, then $H$ splits over $N$.

(ii) If $H$ is a subgroup of $G$, then $\Phi(H)=1$.

(iii) If $P$ is a Sylow p-subgroup of $G$, then $\Phi(P)=1$.

(iv) For each prime $p$, the Sylow p-subgroups of $G$ are elementary Abelian.

Proof. Clearly (i) implies (ii), (ii) implies (iii), and (iii) implies (iv). Then (iv) implies (i) from a result of Gaschütz [3, Theorem 8] that a group 
$G$ whose Sylow $p$-subgroups are all elementary Abelian, splits over each normal subgroup.

Corollary 2.3. (i) A group $G$ is an elementary group if and only if all the Sylow p-subgroups are elementary Abelian p-groups.

(ii) The homomorphic image of an elementary group is an elementary group.

(iii) The direct product of a finite number of elementary groups is an elementary group.

The converse of part (ii) in the corollary is in general not valid but more concerning groups having homomorphic images which are elementary will be discussed in $\$ 4$.

Theorem 2.4. A group $G$ having the property that each proper subgroup is elementary is one of the following types:

(i) $G$ is elementary and not a p-group.

(ii) $G$ is a p-group and

(a) $G$ is an elementary Abelian group, or

(b) $G$ is cyclic of order $p^{2}$, or

(c) $G=\left\{a, b \mid a^{p}=b^{p}=c^{p}=1, c a=a c, c b=b c\right\}$ for an odd prime $p$, or

(d) $G$ is the direct product of a finite number of groups of types (a), (b), or (c).

Proof. If $G$ is not a $p$-group then each Sylow $p$-subgroup is elementary Abelian and consequently $G$ is an elementary group.

If $G$ contains two normal subgroups $M$ and $N$ such that $M \cap N=1$, then $G$ is isomorphic to the direct product of $G / M$ and $G / N$. So it is sufficient to limit the remainder of the discussion to non-Abelian $p$-groups having precisely one minimal normal subgroup contained in $\Phi(G)$. Since the center, $Z(G)$, is prime cyclic, then $Z(G) \leqq \Phi(G)$. Moreover, there must exist at least two maximal subgroups, each elementary Abelian, and thus $[G, G]$ $=\Phi(G)=Z(G)$. Denote a maximal subgroup of $G$ by $H$. Since $\Phi(G) \neq H$, then $G$ contains an element $y, y \notin H$, and $H$ an element $x, x \notin \Phi(G)$, such that $[x, y]=c \neq 1$. $\{x, y\}$ non-Abelian implies $G=\{x, y\}$. Thus the order of $H$ is $p^{2}$ and of $G$ is $p^{3}$ with

$$
G=\left\{x, y \mid x^{p}=y^{p}=c^{p}=1, x c=c x, y c=c y\right\} .
$$

Note that $p$ must be an odd prime for if $p=2$, then $x y=(x y)^{-1}=y^{-1} x^{-1}$ $=y x$.

The study of nonsimple elementary groups is reduced to the study of the semi-direct products of elementary groups. However the converse does not follow, e.g., the symmetric group on four symbols.

THEOREM 2.5. The group $G$ is an elementary group if and only if

(i) $G$ contains a normal elementary subgroup $K$ and an elementary subgroup 
$H$ such that $G=K H, K \cap H=1$, and

(ii) there exists a homomorphism $\theta$ of $H$ into the automorphism group $A(K)$ such that a Sylow p-subgroup of $\mathrm{H \theta}$ leaves elementwise invariant a Sylow psubgroup of $K$ for each prime $p$ dividing the order of $G$.

Proof. Suppose $G$ is an elementary group. Clearly $H$ and $K$ are elementary groups and each Sylow $p$-subgroup $P$ of $G$ is expressible as $P(K) \otimes P(H)$ for some pair of Sylow $p$-subgroups $P(H)$ of $H$ and $P(K)$ of $K$. Hence one can conclude that under the homomorphism $\theta$ of $H$ into $A(K)$, induced out of transformation by elements of $H, P(H)$ is homomorphic to a subgroup $P(H) \theta$ of $A(K)$ which leaves elementwise invariant the elements of $P(K)$. Since under $\theta, P(H) \theta$ is a Sylow $p$-subgroup of $H \theta$, the conditions (i) and (ii) are necessary.

On the other hand, denote by $P(H)$ the Sylow $p$-subgroup of $H$ for which $P(H) \theta$ leaves elementwise invariant a Sylow $p$-subgroup $P(K)$ of $K$ for a fixed prime $p$ and a homomorphism $\theta$ defined as above. Then $P(H)$ lies in the centralizer of $P(K)$ and thus $P=P(K) \otimes P(H)$ is a Sylow $p$-subgroup of $G$. Moreover for this fixed prime $p$, all Sylow $p$-subgroups are conjugate in $G$ and so they are elementary Abelian. Since by (ii) this is true for each prime dividing the order of $G$, the result follows by Corollary 2.3(i).

CoRollary 2.5. If a group $G$ contains a normal subgroup $K$ such that $K$ and $G / K$ are elementary groups of relatively prime orders, then $G$ is elementary.

Several other properties of the elementary groups will be mentioned in brief. As for all $K$-groups, the intersection of the self-normalizing maximal subgroups coincide with the center and hence the centralizer, $Z^{*}(G)$. Moreover, $[G, G] \cap Z^{*}(G)=1$. Whenever the group $G$ is solvable, $G$ is a special case of the $A$-groups of D. R. Taunt and P. Hall [7], [5]. For the supersolvable case the group is Hall-complemented and the structure is well known [5]. The classification of simple elementary groups is incomplete, however it should be noted that of the alternating groups only the one on five symbols is elementary. (A communication from George Glauberman (University of Wisconsin) has indicated an almost complete classification of these from the known simple groups.)

3. $E$-groups. Removing the restriction that $\Phi(G)=1$, one obtains a wellknown property of the nilpotent groups, namely:

Definition. A group $G$ is said to have property $(E)(G \in(E)$ or $G$ is an $E$-group), provided that $\Phi(H) \leqq \Phi(G)$ for each subgroup $H$ of $G$.

TheOREM 3.1. $G \in(E)$ if and only if $G / \Phi(G)$ is an elementary group.

Proof. If $G / \Phi(G)$ is an elementary group, then under the natural homomorphism $\theta$ of $G$ having kernel $\Phi(G),(\Phi(H)) \theta \leqq \Phi(H \theta)=1$, i.e., $\Phi(H)$ 
$\leqq \Phi(G)$ for each subgroup $H$ of $G$.

For the converse note first that with $\theta$ defined as above, $\Phi(G \theta)=1$ since there is a one-to-one correspondence between the maximal subgroups of $G \theta$ and $G$. Then suppose that $W / \Phi(G)$ is isomorphic to a subgroup of $G \theta$, $\Phi(G)<W<G$. Either $\Phi(W / \Phi(G))=1$ or $\Phi(W)$ is properly contained in $\Phi(G)$. Considering the latter, $W$ can be represented as a reduced partial product over $\Phi(G)$ by a subgroup $K$ of $W$, i.e., $W=\Phi(G) K$. Denote by $T$ the subgroup of $W$ for which $\Phi(W / \Phi(G)) \cong T / \Phi(G)$ and suppose there is an element $x \in T \cap K$ such that $x \notin \Phi(G)$. Since $x \notin \Phi(K)$ there then exists a maximal subgroup $S$ of $K$ such that $x \notin S$, and either $\Phi(G) S$ is maximal in $W$ or $W=\Phi(G) S$. The latter contradicts our assumption that $W=\Phi(G) K$ is a reduced partial product. But for the former $T \leqq \Phi(G) S$ which implies that $K=\{S, x\} \leqq \Phi(G) S$ and another contradiction. Thus $\Phi(W)$ must coincide with $\Phi(G)$.

This theorem permits all previous theorems on elementary groups to be applied to the factor group $G / \Phi(G)$ and in particular the characterization of $E$-groups involves the Sylow $p$-subgroups.

TheOREM 3.2. For each prime $p$, the Sylow p-subgroup of $\Phi(G)$ is contained in the normal subgroup $N$ of $G$ of least order which contains $\Phi(P), P a$ Sylow p-subgroup of $G$.

Proof. If $N=1$, then $P$ is an elementary Abelian $p$-group and hence $P$ splits over the Sylow $p$-subgroup $P^{*}$ of $\Phi(G)$. A result of Gaschütz [3, Theorem 1] states that if $A$ is a normal Abelian $p$-subgroup of a group $G$ and $M$ a normal subgroup of $G$ such that $A<M<G,(G: M, A: 1)=1$, then $G$ splits over $A$ if and only if $M$ splits over $A$. Consequently $G$ splits over $P^{*}$. So $P^{*}=1$.

If $N \neq 1, G / N$ has elementary Abelian $p$-subgroups and applying the result for $N=1, N P^{*} \leqq N$, i.e., $P^{*} \leqq N$.

Corollary 3.2. If the group $G$ contains an elementary Abelian Sylow psubgroup for the prime $p, \Phi(G)$ contains no $p$-component.

Theorem 3.3. A necessary and sufficient condition that a group $G$ is an E-group is that $\Phi(P) \leqq \Phi(G)$ for each Sylow p-subgroup $P$ of $G$ and for all primes $p$ dividing the order of $G$.

Proof. If $G \in(E)$ the conditions are clearly necessary. On the other hand it follows from Theorem 3.1 that they are also sufficient since $G / \Phi(G)$ would be elementary.

Corollary 3.3. $G \in(E)$ if and only if $P^{*}$, the Sylow p-subgroup of $\Phi(G)$, is the normal subgroup of least order which contains $\Phi(P), P$ a Sylow $p$ subgroup of $G$, for each prime $p$ dividing the order of $G$. 
Theorem 3.4. For E-groups $A$ and $B$ and a homomorphism $\theta$ of $A$

(i) $A \theta$ and

(ii) $A \otimes B$ are E-groups.

(iii) $(\Phi(A)) \theta=\Phi(A \theta)$.

Proof. Since under a homomorphism $\theta$ of a group $G$ p-groups go onto $p$-groups and also since $(\Phi(G) \theta) \leqq \Phi(G) \theta$, then $A \theta \in(E)$. (ii) follows from the known result [2, Theorem 6] that $\Phi(A \otimes B) \cong \Phi(A) \otimes \Phi(B)$. As for (iii), note that if $N \leqq \Phi(A), N$ the kernel of $\theta$, then $N \leqq \Phi(G)$ since a $G$-normal subgroup $K \leqq \Phi(H), H$ a subgroup of a group $G$, is contained in $\Phi(G)$ $[2$, Theorem 5]. If $N \$ \Phi(A)$ there exists a reduced partial product $A=N K$ such that $\Phi(A / N) \cong N \Phi(K) / N$ by an argument similar to the latter portion of Theorem 3.1, i.e., $\Phi(A \theta)=\Phi(K) \theta$. However, $N \Phi(A) \leqq N \Phi(K) \leqq N \Phi(A)$, the latter since $A \in(E)$, and hence $(\Phi(K)) \theta=(\Phi(A)) \theta=\Phi(A \theta)$.

There also exists another characterization of the $E$-groups in terms of commutators.

Definition. Define $k(G)$ to be the normal subgroup of least order generated by $[\Phi(P), G]$ for each Sylow $p$-subgroup $P$ of a group $G$ and all primes dividing the order of $G$.

TheOREM 3.5. A necessary and sufficient condition that a group $G$ be an E-group is that $k(G) \leqq \Phi(G)$.

Proof. If $G \in(E)$, then $\Phi(P) \leqq \Phi(G)$ and hence the necessity follows.

On the other hand $k(G) \leqq \Phi(G)$ implies $[\Phi(P), G] \leqq \Phi(G)$ for each Sylow $p$-subgroup $P$ of $G$. Next note that $\Phi(P)$ is contained in the intersection, $R$, of the normal maximal subgroups of $G$ for otherwise $G$ contains a normal maximal subgroup $M$ such that $M \cap P$ is a proper subgroup of $P$ and $P$ $=\Phi(P)(M \cap P)$. Moreover, $\Phi(G) \Phi(P) / \Phi(G)$ is contained in $Z(G / \Phi(G))$ $\cong L / \Phi(G)$, where $L$ is the intersection of the self-normalizing maximal subgroups of $G[2$, Theorem 15]. Consequently $\Phi(P) \leqq R \cap L=\Phi(G)$.

Corollary 3.5. For an arbitrary group $G, G / k(G)$ is an E-group.

Proof. Under the natural homomorphism $\theta$ having kernel $k(G), k(G \theta)=1$ since $(k(G)) \theta=k(G \theta)$.

The existence of noncommutative simple elementary groups finds an analogy in the unimodular group $\operatorname{SL}(2,5)$ for $E$-groups. $\Phi(G)$ is nontrivial and $G / \Phi(G)$ is simple.

4. Elementary commutators. In this section an examination will be made of the intersection of the normal subgroups $N$ of a group $G$ having the property that $G / N$ is an elementary group.

Theorem 4.1. Consider a group G.

(i) $G$ contains a unique, characteristic subgroup $E(G)$, the elementary com- 
mutator of $G$, such that $E(G)$ is contained in each normal subgroup $N$ of $G$ for which $G / N$ is elementary.

(ii) $\Phi(G) \leqq E(G) \leqq R, R$ the intersection of the normal maximal subgroups of $G$.

(iii) $E(G)$ is the normal subgroup of least order containing $\Phi(P)$ for all Sylow p-subgroups $P$ of $G$ and each prime $p$ dividing the order of $G$.

(iv) $E(G)$ is the normal subgroup of least order containing $\Phi(H)$ for all subgroups $H \leqq G$.

(v) $G \in(E)$ if and only if $E(G)=\Phi(G)$.

(vi) $E(A \otimes B) \cong E(A) \otimes E(B)$.

(vii) If $G$ is not an elementary group, but every proper homomorphic image of $G$ is an elementary group, then $G$ possesses one and only one minimal normal subgroup.

(viii) $(E(G)) \theta=E(G \theta)$ for a homomorphism $\theta$ of $G$.

Proof. For (i) note that if $U$ and $V$ are normal subgroups of $G$ having the property that $G / U$ and $G / V$ are elementary, then $\Phi(H) \leqq U \cap V$ for each subgroup $H$ of $G$, i.e., $G / U \cap V$ is elementary. From this the existence and uniqueness of $E(G)$ readily follow.

If $M$ is a normal maximal subgroup of a group $G, G / M$ is prime cyclic and by (i), $E(G) \leqq R$. Clearly $\Phi(G) \leqq E(G)$.

For (iii) it is enough to note that for the subgroup $H$ of least order containing $\Phi(P)$ for all Sylow $p$-subgroups $P$ of $G$ and each prime $p$ dividing the order of $G, G / H$ possesses elementary Abelian Sylow $p$-subgroups and hence is elementary.

(iv) is a consequence of (iii) and (v) of (iv).

In (vi) set $G=A \otimes B$. Since the Sylow $p$-subgroups of a direct product is the direct product of the Sylow $p$-subgroups of the factors, then $G /(E(A)$ $\otimes E(B))$ is elementary, i.e., $E(G) \leqq E(A) \otimes E(B)$. However, equality results since under the homomorphism $\theta$, having kernel $E(G)$,

$$
\begin{aligned}
(E(A)) \theta & =(E(B)) \theta \\
& =1 .
\end{aligned}
$$

(vii) is a corollary to a theorem by Baer [1, p. 128] which was proven for more general group properties.

Finally for (viii) one notes that if $\theta$ is a homomorphism of $G, G \theta=H$, $(E(G)) \theta \leqq E(H)$ since $\Phi(K) \theta \leqq \Phi(K \theta)$ for each subgroup $K$ of $G$. However, since $(G / E(G)) \theta$ is an elementary group, $E(H) \leqq(E(G)) \theta$ and equality results.

If $[G, G] \leqq E(G)$, for all groups $G$, then all elementary groups are Abelian. Since this is not the case one can conclude that the derived group is not generally contained in $E(G)$. 


\section{Bibliography}

1. R. Baer, Classes of finite groups and their properties, Illinois J. Math. 1 (1957), 115-187.

2. W. Gaschütz, Uber die \$-Untergruppen endlicher Gruppen, Math. Z. 58 (1953), 160-170.

3. _ Zur Erweiterungtheorie der endlicher Gruppen, J. Reine Angew. Math. 190 (1952), 93-107.

4. M. Hall, The theory of groups, Macmillan, New York, 1959.

5. P. Hall, Complemented groups, J. London Math. Soc. 12 (1937), 201-204.

6. M. Suzuki, Structure of a group and the structure of its lattice of subgroups, Springer, Berlin, 1956.

7. D. R. Taunt, On A-groups, Proc. Cambridge Philos. Soc. 45 (1959), 24-42.

BUCKNELL UNIVERSITY,

Lewisburg, Pennsylvania 